\title{
A Modular Approach to Roberts' Theorem
}

\author{
Shahar Dobzinski * Noam Nisan ${ }^{\dagger}$
}

February 9, 2009

\begin{abstract}
Roberts' theorem from 1979 states that the only incentive compatible mechanisms over a full domain and range of at least 3 are weighted variants of the VCG mechanism termed affine maximizers. Roberts' proof is somewhat "magical" and we provide a new "modular" proof. We hope that this proof will help in future efforts to extend the theorem to non-full domains such as combinatorial auctions or scheduling.
\end{abstract}

\section{Introduction}

Mechanism design theory has gained a place as a conceptual cornerstone for designing computer protocols among self-interested parties, as is found in the internet. For background on mechanism design we refer the reader to standard textbooks in micro-economic theory [10] and for background on its computational applications to part II of [13].

The most basic notion in mechanism design is that of truthfulness in dominant strategies. The setting involves a set of alternatives $A$, and a set of $n$ players, that each has a valuation function $v_{i} \in V_{i} \subseteq \Re^{A}$, where $v_{i}(a)$ denotes the value that player $i$ assigns to alternative $a$. A (direct revelation) mechanism $M=\left(f, p_{1}, \ldots, p_{n}\right)$ contains a preference-aggregation function $f: V_{1} \times \cdots \times V_{n} \rightarrow A$ and payment functions $p_{i}: V_{1} \times \cdots \times V_{n} \rightarrow \Re$. An incentive compatible mechanism ensures that each player's best interest when "reporting" his input $v_{i}$ to the mechanism is to report it truthfully.

Definition 1.1 A mechanism $M=\left(f, p_{1}, \ldots, p_{n}\right)$ is incentive compatible (equivalently truthful or strategyproof) if for all players $i$, all valuations $v_{i}, v_{i}^{\prime} \in V_{i}$ and $v_{-i} \in V_{-i}$,

$$
v_{i}\left(f\left(v_{i}, v_{-i}\right)\right)-p_{i}\left(v_{i}, v_{-i}\right) \geq v_{i}\left(f\left(v_{i}^{\prime}, v_{-i}\right)\right)-p_{i}\left(v_{i}^{\prime}, v_{-i}\right)
$$

We say that $f$ can be implemented if for some $p_{1}, \ldots, p_{n}$, the obtained mechanism is incentive compatible.

As incentive compatibility is the basic requirement in applications, a characterization is of central interest:

Characterization Question: Which preference-aggregation functions $f$ are implementable?

While it is clear why we would like to understand which naturally-desired functions are implementable, in computational applications we require more: understanding the implementability of the family of approximations to a desired function. The necessity of settling for an approximation can be either due to computational hardness of $f$ or due to the unimplementability of $f$ itself.

${ }^{*}$ The School of Computer Science and Engineering, the Hebrew University of Jerusalem. Supported by the Adams Fellowship Program of the Israel Academy of Sciences and Humanities, and by a grant from the Israeli Academy of Sciences. Email: shahard@cs.huji.ac.il.

${ }^{\dagger}$ The Hebrew University of Jerusalem and Google Tel Aviv. Email: noam@cs.huji.ac.il. 
The key positive result in mechanism design, VCG mechanisms (see, e.g., [12]), states that socialwelfare maximization is implementable for every range of alternatives and domain of valuations. I.e., the function $f\left(v_{1}, \ldots, v_{n}\right)=\arg \max _{a} \sum_{i} v_{i}(a)$ is implementable with VCG payments. (In the case that maximum social welfare is obtained at more than alternative, any of them can be chosen.) It is easy to generalize the VCG mechanisms to weighted variations, termed affine maximizers:

Definition $1.2 f: V_{1} \times \cdots \times V_{n} \rightarrow A$ is an affine maximizer if there exist real constants $\alpha_{1}, \ldots, \alpha_{n}$, $\alpha_{i} \geq 0$, and $\beta_{a}$ for all $a \in A$ such that for all $v, f(v) \in \arg \max _{a} \sum_{i}\left(\alpha_{i} v_{i}(a)\right)+\beta_{a}$.

The main impossibility result in the area is the surprising theorem of Roberts:

Theorem $1.3([\mathbf{1 5}])$ : The only implementable functions with range of size more than $2,|A| \geq 3$ and full domains $V_{i}=\Re^{A}$ are affine maximizers.

The main restriction in the theorem is the requirement of full domain. The extreme opposite case is where the domain is essentially single dimensional. In these cases, termed "single-parameter", much more can be implemented, and a good characterization is known (see, e.g., [12]), which has been used extensively in computational settings $[1,9,4]$. The case of $|A|=2$ is always single parameter, which explains why the theorem required $|A| \geq 3$.

Most interesting computational applications do not have a full domain nor are they single-parameter, and indeed we do not have good characterizations of which functions are implementable for, e.g., combinatorial auctions, multi-unit auctions, or scheduling. All of the few such characterizations known are in very restricted settings: restricted auction domains [7,6] and "combinatorial public projects" [14]. All these papers start by showing that all implementable mechanisms are affine maximizers by proving Roberts-like theorems, and then provide lower bounds on what can be achieved by affine-maximizers in polynomial time [5]. This lack of characterization is the underlying reason for the very little progress on the long standing problems of how well can computationally-efficient incentive compatible mechanisms approximate the optimal allocation in combinatorial auctions, or approximate the optimal schedule in scheduling problems. Of note is a positive result for "combinatorial auctions with duplicate items" [3].

Extending Roberts' theorem to other domains has remained elusive. While Roberts' proof itself is not very difficult or long, it is quite mysterious (to us, at least). There is no clear separation into independent tasks, each which can be extended (or not) to non-full domains. The second author has already been involved in efforts to extend [7] and simplify the proof of [8] Roberts' theorem, but still finds it mysterious. During the last few years the two authors have spend considerable time in attempting to extend - or at least obtain a really clear proof of - Roberts' theorem. While we can not claim to be completely satisfied, we feel that we do have a new modular proof that may be of interest and so we bring it here. From a technical point of view, our proof is completely combinatorial and does not rely on the separation theorem between convex bodies, unlike the original proof.

Here is a high-level structure of the new proof.

1. We begin with the direct and standard [12] observations:

- An implementable $f$ is "weakly monotone": $f\left(v_{i}, v_{-i}\right)=a$ and $f\left(v_{i}^{\prime}, v_{-i}\right)=b$ implies that $v_{i}(a)-v_{i}(b) \geq v_{i}^{\prime}(a)-v_{i}^{\prime}(b)$.

- The payment function for player $i$ does not depend on $v_{i}$, and may be represented as $p_{i}\left(v_{i}, v_{-i}\right)=$ $p_{i}^{a}\left(v_{-i}\right)$ for $a=f\left(v_{i}, v_{-i}\right)$.

- $f$ must optimize for each player: $f\left(v_{i}, v_{-i}\right) \in \arg \max _{a} v_{i}(a)-p^{a}\left(v_{-i}\right)$.

2. The next step is due to [7] and shows that "ties can be ignored". Specifically, we may assume without loss of generality that the " $\geq$ " in the weak monotonicity condition is in fact strict, a condition termed strong monotonicity: $f\left(v_{i}, v_{-i}\right)=a$ and $f\left(v_{i}^{\prime}, v_{-i}\right)=b$ implies that $v_{i}(a)-v_{i}(b)>$ $v_{i}^{\prime}(a)-v_{i}^{\prime}(b)$. As shown in [7] a the critical element here is full-dimensionality of the domains. 
3. The third step is a proof that $f$ must have a player with no veto power - i.e., that for every value of $v_{i}$, the range of $f$ after fixing this value still remains full. This is somewhat in spirit of Barbera and Peleg's proof [2] of Gibbard-Satterthwaite theorem. A critical element is the un-boundedness of the valuation space, shedding some light on the bounded domain example of [11].

4. The fourth step in the proof is the case $n=2$ (when we already know that there is a player with no veto power). Here we observe that that pricing functions $p_{i}^{a}$ mentioned in step 1 satisfy a simple condition that $p_{i}^{a}\left(v_{1}\right)-p_{i}^{b}\left(v_{1}\right)$ is a (monotone) function only of the scalar $v_{1}(a)-v_{1}(b)$. Simple closed mathematical reasoning implies that real functions that satisfy these conditions must all be linear with the same slope, which directly proves the statement. The critical element in this argument is that the range for every fixed value of $v_{1}$ is of size at least 3 .

5. The fifth step is induction on $n$, with the base step being at $n=2$. The logic is basically that every restriction of a single player results in prices that are linear functions of the remaining players, and since it can be shown that the slopes must be equal for different restrictions, we get the prices must be linear over-all.

\section{Preliminaries}

We start with some notation. $A$ is the set of alternatives, $|A| \geq 3 . V=\Re^{A}$ is the full domain of valuations of a single player. An $n$-player mechanism is a pair $(f, p)$ where $f: V^{n} \rightarrow A$ and $p: V^{n} \rightarrow \Re$, and $p=\left(p_{1}, \cdots, p_{n}\right)$, where $p_{i}: V \rightarrow R$.

Definition 2.1 $v^{a+=\delta}$ the valuation obtained from $v$ by increasing the value for a by $\delta$ and not changing any other value.

In the rest of the paper, unless noted otherwise, $i$ will range over $1, \ldots, n ; a, b, c, k$ will always range over $A, v_{i}, v_{i}^{\prime}$ will always range over $V$, and $v_{-i}$ will range over $V^{n-1}$.

Definition $2.2(f, p)$ is incentive compatible if for all $i$, all $v_{i}, v_{i}^{\prime}$ and all $v_{-i}$ we have that $v_{i}\left(f\left(v_{i}, v_{-i}\right)\right)-$ $p\left(v_{i}, v_{-i}\right) \geq v_{i}^{\prime}\left(f\left(v_{i}^{\prime}, v_{-i}\right)\right)-p\left(v_{i}^{\prime}, v_{-i}\right)$.

The next two propositions are standard, and hold over all domains not just the full domain:

Definition $2.3 f$ is weakly monotone if for all $v_{i}, v_{i}^{\prime}$ and $a, b: f\left(v_{i}, v_{-i}\right)=a$ and $f\left(v_{i}^{\prime}, v_{-i}\right)=b$ implies $v_{i}(a)-v_{i}(b) \geq v_{i}^{\prime}(a)-v_{i}^{\prime}(b)$.

Lemma 2.4 (e.g., [12]) If $(f, p)$ is incentive compatible then there exist functions $p_{i}^{a}: V^{n-1} \rightarrow \Re \cup$ $\{\infty\}$ such that

1. Whenever $f\left(v_{i}, v-i\right)=a$ we have that $p_{i}\left(v_{i}, v_{-i}\right)=p^{a}\left(v_{-i}\right)$.

2. $f\left(v_{i}, v_{-i}\right) \in \arg \max _{a} v_{i}(a)-p_{i}^{a}\left(v_{-i}\right)$.

Lemma 2.5 ([7]) If $(f, p)$ is incentive compatible then $f$ is weakly monotone.

Proof: Since $f$ is incentive compatible, we have that $v_{i}(a)-p_{i}^{a}\left(v^{-i}\right) \geq v_{i}(b)-p_{i}^{b}\left(v^{-i}\right)$, since $f\left(v_{i}, v_{-i}\right)=$ $a$. On the other hand, $f\left(v_{i}^{\prime}, v_{-i}\right)=b$, and thus $v_{i}^{\prime}(b)-p_{i}^{b}\left(v^{-i}\right) \geq v_{i}^{\prime}(a)-p_{i}^{a}\left(v^{-i}\right)$. Subtracting the two inequalities we get that $v_{i}(a)-v_{i}(b) \geq v_{i}^{\prime}(a)-v_{i}^{\prime}(b)$.

Definition 2.6 $f$ is an affine maximizer if there exists constants $\alpha_{i} \geq 0$ and $\beta_{a} \in \Re \cup\{\infty\}$ such that $f\left(v_{1}, \ldots, v_{n}\right) \in \arg \max _{a}\left(\sum_{i}\left(\alpha_{i} v_{i}(a)\right)-\beta_{a}\right)$. 


\section{Getting Rid of Ties}

Definition $3.1 f$ is strongly monotone if for all $v_{i}, v_{i}^{\prime}$ and $a, b: f\left(v_{i}, v_{-i}\right)=a$ and $f\left(v_{i}^{\prime}, v_{-i}\right)=b$ implies $v_{i}(a)-v_{i}(b)>v_{i}^{\prime}(a)-v_{i}^{\prime}(b)$.

Lemma 3.2 ([7]) : If for every incentive compatible $(f, p)$ with a strongly monotone $f, f$ is an affine maximizer, then also for every incentive compatible $(f, p), f$ is an affine maximizer.

\section{Existence of No-Veto-Power Players}

Definition 4.1 Player $i$ is said to hold no veto power in $f$ if for every $v_{i}$ and every a there exists $v_{-i}$ with $f\left(v_{i}, v_{-i}\right)=a$. Player $i$ said to be decisive in $f$ if for every $v_{-i}$ and for every a there exists some $v_{i}$ such that $f\left(v_{i}, v_{-i}\right)=a$.

Lemma 4.2 If $(f, p)$ is incentive compatible and $f$ is strongly monotone then all players, except perhaps a single one, hold no veto power.

We will prove this by considering the range of $v_{i}$ (for some fixed player $i$ ):

Definition 4.3 range $\left(v_{i}\right)=\left\{f\left(v_{i}, v_{-i}\right)\right\}_{v_{-i}}$.

Lemma 4.4 If $(f, p)$ is incentive compatible and $f$ is strongly monotone and onto $A$ then range $(\cdot)$ satisfies the following properties:

1. Full Range: $\cup_{v_{i}}$ range $\left(v_{i}\right)=A$.

2. Monotonicity: $a \in \operatorname{range}\left(v_{i}\right)$ and $\delta \geq 0$ implies $a \in \operatorname{range}\left(v_{i}^{a+=\delta}\right)$.

3. IIA : $v_{i}(a)-v_{i}(b)=v_{i}^{\prime}(a)-v_{i}^{\prime}(b)$ implies that range $\left(v_{i}\right) \cap\{a, b\}=\operatorname{range}\left(v_{i}^{\prime}\right) \cap\{a, b\}$ or range $\left(v_{i}\right) \cap$ $\{a, b\}=\emptyset$.

Proof: We first show that $f$ has a full range. This follows immediately from $f$ being onto $A$ : for each alternative $a$, let $v_{i}^{a}, v_{-i}^{a}$ be so that $f\left(v_{i}^{a}, v_{-i}^{a}\right)=a$. $a \in \operatorname{range}\left(v_{i}^{a}\right)$, and thus $\cup_{a \in A} \operatorname{range}\left(v_{i}^{a}\right)=A$.

For monotonicity, consider $v_{i}$ such that $a \in \operatorname{range}\left(v_{i}\right)$, and $v_{-i}$ be such that $f\left(v_{i}, v_{-i}\right)=a$. By strong monotonicity, for every $\delta>0, f\left(v_{i}^{a+=\delta}, v_{-i}\right)=a$. Hence $a \in \operatorname{range}\left(v_{i}^{a+=\delta}\right)$.

The proof of IIA is a bit more involved. Let $v_{i}, v_{i}^{\prime}$ be as in the IIA condition. It is enough to show that it cannot be the case the $a, b \in \operatorname{range}\left(v_{i}\right), a \in \operatorname{range}\left(v_{i}^{\prime}\right)$, but $b \notin \operatorname{range}\left(v_{i}^{\prime}\right)$. Suppose not. Consider the case where the valuation of each of the other players is identical and defined as follows: $u(b)=0$, $u(a)=t$, and for each other alternative $k \neq a, b, u(k)=-t$, for some $t$ to be defined later. We will show that $f(v, u, \ldots, u)=a$ and $f\left(v^{\prime}, u, \ldots, u\right)=b$, and obtain a contradiction to strong monotonicity.

We start by showing that $f(v, u, \ldots, u)=a$. Since $a \in \operatorname{range}(v)$, there exist valuations $u_{2}^{\prime}, \ldots, u_{n}^{\prime}$ such that $f\left(v, u_{2}^{\prime}, \ldots, u_{n}^{\prime}\right)=a$. Choose $t$ to be large enough, so that for every $i \geq 2$, and alternative $k \neq$ $a, b: t-u_{i}^{\prime}(a)=u(a)-u_{i}^{\prime}(a) \geq \max _{k} u(k)-u_{i}^{\prime}(k)$. By strong monotonicity we have that $f(v, u, \ldots, u)=a$.

We now show that $f\left(v^{\prime}, u, \ldots, u\right)=b$. Since $b \in \operatorname{range}\left(v^{\prime}\right)$, there exist valuations $u_{2}^{\prime}, \ldots, u_{n}^{\prime}$ such that $f\left(v^{\prime}, u_{2}^{\prime}, \ldots, u_{n}^{\prime}\right)=b$. Choose $t$ to be large enough so that: $0-u_{i}^{\prime}(b)=u(b)-u_{i}^{\prime}(b) \geq \max u(k)-u_{i}^{\prime}(k)=$ $-t-u_{i}^{\prime}(k)$, for every alternative $k \neq a$ and $i \geq 2$. We have that $f\left(v^{\prime}, u, \ldots, u\right) \in\{a, b\}$. However, $a \notin \operatorname{range}\left(v^{\prime}\right)$, and thus $f\left(v^{\prime}, u, \ldots, u\right)=b$, as needed.

The rest of the proof considers any $R(\cdot)$ that satisfies these three conditions.

Definition 4.5 Alternative $a$ is dictatable in $R: V \rightarrow 2^{A} \backslash\{\emptyset\}$ if for some $v, R(v)=\{a\}$. 
Lemma 4.6 If $R: V \rightarrow 2^{A} \backslash\{\emptyset\}$ satisfies Full Range (for $|A| \geq 3$ ), Monotonicity, and IIA then either all alternatives are dictatable in $R$ or none are.

Proof: The proof consists of the following series of claims.

Claim 4.7 For all $v, a, \delta>0$, either $R\left(v^{a+=\delta}\right)=\{a\}$ or $R(v) \subseteq R\left(v^{a+=\delta}\right) \cup\{a\}$.

Proof: We show that no alternative is removed from the $R\left(v^{a+=\delta}\right)$ unless $a$ remains alone in the range. Assume $b \neq a$ remained in $R\left(v^{a+=\delta}\right)$, and that $c \neq b, a$ was removed. However, this is a contradiction to IIA, since $v(b)-v(c)=v^{a+=\delta}(b)-v^{a+=\delta}(c)$.

Claim 4.8 For all $v$ and for all alternatives $a$, there exists some $\delta>0$ such that $a \in R\left(v^{a+=\delta}\right)$.

Proof: Let $v^{\prime}$ be such that $a \in R\left(v^{\prime}\right)$. Fix some $b \in R(v)$. Assume without loss of generality $v^{\prime}(a)-v^{\prime}(b) \geq v(a)-v(b)$ (else, consider $v^{a+=\gamma}$ instead of $v^{\prime}$, for sufficiently large $\gamma>0$, and still have $a \in R\left(v^{\prime a+=\gamma}\right)$ by monotonicity). Let $\delta=v^{\prime}(a)-v^{\prime}(b)-(v(a)-v(b))$. By Claim 4.7 either $a \in R\left(v^{a+=\delta}\right)$ (and we are done), or $b \in R\left(v^{a+=\delta}\right)$ (since $R(v) \subseteq R\left(v^{a+=\delta}\right)$ ). In the latter case, observe that since $v^{\prime}(a)-v^{\prime}(b)=v^{a+=\delta}(a)-v^{a+=\delta}(b)$, by IIA and since $b \in R\left(v^{a+=\delta}\right)$ we also have that $a \in R\left(v^{a+=\delta}\right)$.

Claim 4.9 Let $a$ be a non dictatable alternative. Let $v$ be such that $a, b \in R(v)$. Let $w$ be such that $v(a)-v(b) \leq w(a)-w(b)$. Then, if $a \in R(w)$ we also have that $b \in R(w)$.

Proof: Let $\delta=w(a)-w(b)-(v(a)-v(b))$. By Claim 4.7, $a, b \in R\left(v^{a+=\delta}\right)$ (since $a$ is non-dictatable). The claim now follows by using IIA.

Claim 4.10 Let a be a non-dictatable alternative. For all $v$, there exists $\delta>0$ such that $R\left(v^{a+=\delta}\right)=A$.

Proof: For alternative $k$, let $w_{k}$ be a valuation with $a, k \in \operatorname{range}(w)$. Such a valuation exists: Let $w_{k}^{\prime}$ be such that $k \in R\left(w_{k}^{\prime}\right)$. By Claim 4.8, for some $\delta_{k}>0, a \in R\left(w_{k}^{a+=\delta_{k}}\right)$. By Claim 4.7, $k \in R\left(w_{k}^{a+=\delta_{k}}\right)$.

Fix $v$. For each $k$, let $r_{k}=w_{k}(a)-w_{k}(k)$. Let $\gamma>0$ be so that $a \in R\left(v^{a+=\gamma}\right)$, as guaranteed from Claim 4.8. Let $\delta \geq \gamma$ be such that $v^{a+=\delta}(a)-v^{a+=\delta}(a) \geq w_{k}(a)-w_{k}(k)$, for every $k$. By Claim 4.7 $a \in R\left(v^{a+=\delta}\right)$. By Claim $4.9 k \in R\left(v^{a+=\delta}\right)$, for all $k$.

To finish the proof of Lemma 4.6, suppose there is a dictatable alternative $a$, and a non-dictatable one $b$. Let $v, \delta>0$ be such that $R(v)=\{a\}$, and $R\left(v^{b+=\delta}\right)=A$ (as guaranteed by Claim 4.10). However, for $c \neq b, a$ we have that $v(a)-v(c)=v^{b+=\delta}(a)-v^{b+=\delta}(c)$. By IIA $b \in R(v)$. A contradiction.

Lemma 4.11 If all alternatives of a player are non-dictatable then the player holds no veto power.

The lemma immediately gives us Lemma 4.2 since at most one player can have all his alternatives dictatable (otherwise two players will dictate contradicting alternatives).

Proof: Let $v$ be some valuation. Let $a, b$ be some alternatives with $a, b \in R(v)$ (the existence of two such alternatives is guaranteed since all alternatives are non dictatable). By Claim 4.10, there is some $\delta>0$ such that $R\left(v^{a+=\delta}\right)=A$. For every other $c \neq a, b$, we have that $v(b)-v(c)=v^{a+=\delta}(b)-v^{a+=\delta}(c)$, and thus, by IIA and since $R\left(v^{a+=\delta}\right)=A$, we also have that $c \in R(v)$, and hence $R(v)=A$. 


\section{Two Players}

Lemma 5.1 Let $f: V^{2} \rightarrow A$. If $(f, p)$ is incentive compatible, $f$ satisfies strong monotonicity, and the second player is decisive then $f$ is an affine maximizer.

Definition $5.2 p: \Re^{m} \rightarrow \Re^{m}$ is pair-wise-determined if $x_{a}-x_{b}=x_{a}^{\prime}-x_{b}^{\prime}$ implies $p^{a}(x)-p^{b}(x)=p^{a}\left(x^{\prime}\right)-$ $p^{b}\left(x^{\prime}\right)$. It is pair-wise-monotone (decreasing) if $x_{a}-x_{b}>x_{a}^{\prime}-x_{b}^{\prime}$ implies $p^{a}(x)-p^{b}(x) \leq p^{a}\left(x^{\prime}\right)-p^{b}\left(x^{\prime}\right)$.

Claim 5.3 If $(f, p)$ is incentive compatible, $f$ satisfies strong monotonicity, and the second player is decisive, then the vector $p$ of payment functions $p^{a}: \Re^{m} \rightarrow \Re$ associated with it by Lemma 2.4 is pair-wise-determined and pair-wise-monotone.

Proof: We first note that the function $p^{a}(x)$ are always finite, as an infinite value of $p^{a}(x)$ will cause $a$ never to be the value of $f$ contradicting decisiveness. Now assume by way of contradiction to one of these assertions that $p^{a}(x)-p^{b}(x)>p^{a}\left(x^{\prime}\right)-p^{b}\left(x^{\prime}\right)$, while $x_{a}-x_{b} \geq x_{a}^{\prime}-x_{b}^{\prime}$. Choosing $y$ such that $p^{a}(x)-p^{b}(x)>y_{a}-y_{b}>p^{a}\left(x^{\prime}\right)-p^{b}\left(x^{\prime}\right)$, with low values for all other $y_{c}$ 's will give, by Lemma 2.4, $f(x, y)=b$ but $f\left(x^{\prime}, y\right)=a$, contradicting strong monotonicity.

The rest of the proof follows directly from this property:

Claim 5.4 Let $p: \Re^{m} \rightarrow \Re^{m}$ be pair-wise determined and pair-wise-monotone then for some fixed function $h: \Re^{m} \rightarrow \Re$, constant $\alpha \geq 0$ and constants $\beta_{a} \in \Re$ we have that for all $a, p^{a}(x)=h(x)-\alpha x_{a}-\beta_{a}$.

Claim 5.4 directly implies the lemma: By Lemma 2.4, we know that $f(x, y) \in \arg \max _{a}\left(y_{a}-p^{a}(x)\right)$ and since $h(x)$ does not depend on $a, f(x, y) \in \arg \max _{a}\left(y_{a}+\alpha x_{a}+\beta_{a}\right)$ as required.

Proof: (of Claim 5.4) For ease of notation, we start by assuming without loss of generality that $p^{c}(x)=0$ for all $x$, where $c$ is some fixed alternative. This is without loss of generality since neither the assumptions nor the result of the lemma changes when subtracting a fixed function $p^{c}$ from all entries $p^{a}$. It now suffices to prove the characterization for $x$ with that $x_{c}=0$ since by pair-wise determination adding a constant $k$ to all entries does not change $p^{a}(x)$, while increasing the right-hand-side by the fixed constant $\alpha \cdot k$ (the same for all $a$ ) which can be folded back into $h(x)$.

Definition $5.5 \Delta^{a}(x)=p^{a}\left(x^{a+=\delta}\right)-p^{a}(x)$.

Claim 5.6 For every $x, \Delta^{a}(x)=\Delta^{b}(x)$.

Proof: By pair-wise determination applied to $a, c$ we have that $p^{a}\left(x^{a+=\delta}\right)=p^{a}\left(x^{a+=\delta, b+=\delta}\right)$ and similarly $p^{b}\left(x^{b+=\delta}=p^{b}\left(x^{a+=\delta, b+=\delta}\right)\right.$. But then

$$
\begin{gathered}
\Delta^{a}(x)-\Delta^{b}(x)=\left(p^{a}\left(x^{a+=\delta}\right)-p^{a}(x)\right)-\left(p^{b}\left(x^{b+=\delta}\right)-p^{b}(x)\right)= \\
\left(p^{a}\left(x^{a+=\delta, b+=\delta}\right)-p^{b}\left(x^{a+=\delta, b+=\delta}\right)-\left(p^{a}(x)-p^{b}(x)\right)=0\right.
\end{gathered}
$$

where the equality to 0 follows from pair-wise determination applied to $a, b$.

Claim 5.7 There exists a constant $l=l(\delta)$ such that for all $x$ and $a, \Delta^{a}(x)=l(\delta)$.

Proof: Using pair-wise determination on $a, c, \Delta^{a}(x)$ may only depend on $x_{a}-x_{c}$, and similarly $\Delta^{b}(x)$ may only depend on $x_{b}-x_{c}$. Since Claim 5.6 showed that these are equal then for all $x, y$ such that $x_{c}=y_{c}$ also $\Delta^{a}(x)=\Delta^{a}(y)$. Now take $x, y$ - by pair-wise determination $\Delta^{a}(x)=\Delta^{a}\left(y^{a+=y_{c}-x_{c}}\right)=\Delta^{a}(y)$, where the last equality is since $y$ and $y^{a+=y_{c}-x_{c}}$ have the same $c$-coordinate.

We now conclude the proof of Claim 5.4. By definition we have that $l(\delta+\gamma)=l(\delta)+l(\gamma)$ and so for integer $k, l(k \delta)=k \cdot l(\delta)$, and then also for rational $q, l(q \delta)=q \cdot l(\delta)$. By the definition of pairwise (decreasing) monotonicity (used here for the only time) applied to $a, c$ we see that $\delta \geq \gamma$ implies $l(\delta) \leq l(\gamma)$. This implies the extension of $l(q \delta)=q \cdot l(\delta)$ to all reals $q$. Now define $\alpha=-l(1)$ (with $\alpha \geq 0$ since $l(1)<0)$ and we have that for every $x, y$ and $a, p^{a}(x)-p^{a}(y)=-\alpha \cdot\left(x_{a}-y_{a}\right)$. Now define $\beta_{a}=-p^{a}(\overrightarrow{0})$ so $p^{a}(x)=-\alpha \cdot x_{a}-\beta_{a}$ as required. 


\section{$6 \quad n \geq 3$ Players}

Theorem 6.1 ([15]) Let $(f, p)$ be incentive compatible and onto then $f$ is an affine maximizer.

Proof: By Lemma 3.2 we assume with out loss of generality that $f$ is strongly monotone. We now prove the result by induction on $n$, with our base case $n=2$ shown in Lemma 5.1.

Assume correctness for $n-1$ players. We now prove for $n$. By Lemma 4.2, all players, except perhaps player $n$ (without loss of generality) have no veto power, and thus for any fixed value of $v_{1}$, the induced function, $f_{v_{1}}\left(v_{-1}\right)=f\left(v_{1}, v_{-1}\right)$, is onto so by the induction hypothesis is an affine maximizer $f_{v_{1}}\left(v_{-1}\right) \in \arg \max _{a}\left(\sum_{i}\left(\alpha_{i}^{v_{1}} v_{i}(a)\right)-\beta_{a}^{v_{1}}\right)$. Without loss of generality we assume that $f_{v_{1}}$ is normalized: for each $v_{1}$ and all $i, \alpha_{i} \leq 1$, with at least one $\alpha_{1}^{v_{1}}=1$, and that we have $\beta_{c}^{v_{1}}=0$. We now show:

Lemma 6.2 The values $\alpha_{i}$ do not depend on $v_{1}$. I.e., for each $v_{1}$ and $v_{1}^{\prime}$ and $i, \alpha_{i}^{v_{1}}=\alpha_{i}^{v_{1}^{\prime}}$.

Proof: Suppose not. Without loss of generality, $v_{1}$ and $v_{1}^{\prime}$ differ only in their value for $c$, and $\alpha_{i}^{v_{1}}>\alpha_{i}^{v_{1}^{\prime}}$. Let $j$ be the player with $\alpha_{j}^{v_{1}}=1$. Observe that $\alpha_{j}^{v_{1}} \leq \alpha_{j}^{v_{1}^{\prime}}$, since the weights are normalized. Define the following valuations: for player $j, v_{j}(a)=t$, where $t>>\left|\beta_{a}^{v_{1}}-\beta_{b}^{v_{1}}\right|$, and $v_{j}(k)=0$ for all $k \neq a$. For player $i$, define $v_{i}(b)=\left(\alpha_{j}^{v_{1}} v_{j}(b)+\left(\beta_{a}^{v_{1}}-\beta_{b}^{v_{1}}\right)-\epsilon\right) / \alpha_{i}$, and $v(k)=0$ for all $k \neq b$. For each player $l \neq 1, j, k$ let $v_{l}$ be identically zero. For small enough values of $\epsilon>0$, we have that $f_{v_{1}}\left(v_{1}, v_{2}, \ldots, v_{n}\right)=a$ but $f_{v_{1}}\left(v_{1}^{\prime}, v_{2}, \ldots, v_{n}\right)=b$, a contradiction to strong monotonicity.

If there is a player with $\alpha_{i}=0$, then the output does not depend on his valuation. In this case $f$ is essentially a function for $n-1$ players, and hence it is an affine maximizer by the induction hypothesis. Else, all $\alpha_{i}>0$, and in particular we have that all players, perhaps except the first one, are decisive.

Lemma 6.3 For each $v_{1}, v_{1}^{\prime}, \beta_{a}^{v_{1}}-\beta_{a}^{v_{1}^{\prime}}=\alpha_{1}\left(v_{1}(a)-v_{1}^{\prime}(a)\right)$, for every alternative a.

Proof: We require the following claim first:

Claim 6.4 $\beta_{a}^{v_{1}}$ depends only on $v_{1}(a)$.

Proof: Let $v_{1}, v_{1}^{\prime}$ be such that $v_{1}(a)=v_{1}^{\prime}(a)$ and $\beta_{a}^{v_{1}}>\beta_{a}^{v_{1}^{\prime}}$. Let $v_{2}(a)=-\left(\beta_{a}^{v_{1}}-\beta_{a}^{v_{1}^{\prime}}\right) / 2, v_{2}(c)=0$, and for each other $k \neq a, c, v(k)=-\max \left(\left|\beta_{k}^{v_{1}}\right|,\left|\beta_{k}^{v_{1}^{\prime}}\right|\right)-\epsilon$, for some $\epsilon>0$. Let all other valuations be identically zero. Now, $f\left(v_{1}, v_{-i}\right)=a$ while $f\left(v_{1}^{\prime}, v_{-i}\right)=c$, a contradiction to strong monotonicity.

Thus, it is enough to consider identical valuations $v_{1}, v_{1}^{\prime}$ that only differ in their value for $a$. Consider the following two ways to calculate the output. In the first one, given valuations $v_{1}, \ldots, v_{n}$ we calculate the output according to $f_{v_{1}}$. In the second one, define $f_{v_{2}, \ldots, v_{n-1}}\left(v_{1}, v_{n}\right)=f\left(v_{1}, \ldots, v_{n}\right)$ and calculate according to $f_{v_{2}, \ldots, v_{n-1}}$. Since fixing some players and using the same price functions still result in a truthful mechanism, we may assume that in both $f_{v_{2}, \ldots, v_{n-1}}$ and $f_{v_{1}}$ the prices are calculated according to the price functions $p_{1}, \ldots, p_{n}$ of $f$. Also notice that by the induction hypothesis both functions are affine maximizers (the range of both is $A$ since player $n$ is decisive). Now, for each alternative $a$ :

$$
p_{i}^{a}\left(v_{1}\right)-p_{i}^{a}\left(v_{1}^{\prime}\right)=\alpha_{1}\left(v_{1}(a)-v_{1}^{\prime}(a)\right)=\Sigma_{i \geq 2} \alpha_{i} v_{i}(a)+\beta_{a}^{v_{1}}-\left(\Sigma_{i \geq 2} \alpha_{i} v_{i}(a)+\beta_{a}^{v_{1}^{\prime}}\right)=\beta_{a}^{v_{1}}-\beta_{a}^{v_{1}^{\prime}}
$$

where the first equality is by calculating the price difference according to $f_{v_{2}, \ldots, v_{n-1}}$ and using the fact that it is an affine maximizer, the second equality is by calculating the price difference according to $f_{v_{1}}$ and $f_{v_{1}^{\prime}}$ and taking into account that both $f_{v_{1}}$ and $f_{v_{1}^{\prime}}$ are affine maximizers.

In total we get that the function $f_{v_{1}}$ maximizes a function of the form $\arg \max _{a} \Sigma_{i \geq 2} \alpha_{i} v_{i}(a)+\left(\beta_{a}+\right.$ $\left.\alpha_{1} v(a)\right)=\arg \max _{a} \Sigma_{i \geq 1} \alpha_{i} v_{i}(a)+\beta_{a}$, hence $f$ is an affine maximizer, as needed. 


\section{Acknowledgements}

We thank Itai Ashlagi, Ron Lavi, Dov Monderer, and Ariel Procaccia for helpful discussions.

\section{References}

[1] Aaron Archer and Éva Tardos. Truthful mechanisms for one-parameter agents. In FOCS'01.

[2] Salvador Barbera and Bezalel Peleg. Strategy-proof voting schemes with continuous preferences. Social Choice and Welfare, 1988.

[3] Yair Bartal, Rica Gonen, and Noam Nisan. Incentive compatible multi unit combinatorial auctions. In $T A R K 03$.

[4] Peerapong Dhangwatnotai, Shahar Dobzinski, Shaddin Dughmi, and Tim Roughgarden. Truthful approximation schemes for single-parameter agents. In FOCS'08.

[5] Shahar Dobzinski and Noam Nisan. Limitations of vcg-based mechanisms. In STOC'07.

[6] Shahar Dobzinski and Mukund Sundararajan. On characterizations of truthful mechanisms for combinatorial auctions and scheduling. In EC'08.

[7] Ron Lavi, Ahuva Mu'alem, and Noam Nisan. Towards a characterization of truthful combinatorial auctions. In FOCS'03.

[8] Ron Lavi, Ahuva Mualem, and Noam Nisan. Two simplified proofs for roberts theorem. Social Choice and Welfare, 2009.

[9] Daniel Lehmann, Liadan Ita O'Callaghan, and Yoav Shoham. Truth revelation in approximately efficient combinatorial auctions. In JACM 49(5), pages 577-602, Sept. 2002.

[10] A. Mas-Collel, W. Whinston, and J. Green. Microeconomic Theory. Oxford university press, 1995.

[11] Benny Moldovanu and Moritz Meyer ter Vehn. Ex-post implementation with interdependent valuations. Technical report, 2002.

[12] Noam Nisan. 2007. Introduction to Mechanism Design (for Computer Scientists). In "Algorithmic Game Theory", N. Nisan, T. Roughgarden, E. Tardos and V. Vazirani, editors.

[13] Noam Nisan, Tim Roughgarden, Eva Tardos, and Vijay V. Vazirani. Algorithmic Game Theory. Cambridge University Press, New York, NY, USA, 2007.

[14] Christos Papadimitriou, Michael Schapira, and Yaron Singer. On the hardness of being truthful. In FOCS, 2008.

[15] Kevin Roberts. The characterization of implementable choise rules. In Jean-Jacques Laffont, editor, Aggregation and Revelation of Preferences. Papers presented at the first European Summer Workshop of the Economic Society, pages 321-349. North-Holland, 1979. 